\title{
A131 INCREASED APOPTOSIS IN LYMPHOID CELLS MAY BE THE INITIAL TRIGGER IN THE PATHOGENESIS OF PRISTANE-INDUCED ARTHRITIS
}

S Herman, ${ }^{1} \mathrm{~J}$ Pfatschbacher, ${ }^{1}$ A Fischer, ${ }^{1} \mathrm{M}$ Hoffmann, ${ }^{2} \mathrm{G}$ Steiner ${ }^{1}$ 'Department of Rheumatology, Medical University of Vienna, Vienna, Austria; ${ }^{2}$ Division of Medical Inflammation Research, Karolinska Institutet, Stockholm, Sweden

\subsection{6/ard .2010.148999.7}

Background Increased apoptosis/necrosis and/or deficient clearance of apoptotic material may lead to the release of potential autoantigens provoking the break of self-tolerance mechanisms which may eventually lead to autoimmune diseases such as rheumatoid arthritis (RA). To gain more insight in the role of apoptotic processes in the generation of arthritogenic autoantigens the authors are studying autoimmune responses in the pristane-induced arthritis (PIA) model which shows some striking similarities with human RA including the presence of rheumatoid factor and autoantibodies as well as autoreactive $T$ cells against heterogeneous nuclear ribonucleoprotein A2 (RA33).

Objective To gain more insight in the earliest pathogenetic processes of PIA with special regard to apoptosis/necrosis and consecutive generation of autoantigens and their involvement in arthritis development.

Methods Pristane (2,6,10,14-tetramethylpentadecane) was injected subcutanously at the base of the tail in DA.1F rats. Apoptosis/necrosis rates in lymph nodes and blood were analysed at various time points after pristane application by immunhistochemistry and flow cytometry. Furthermore, phagocytosis efficiency of peripheral blood cells was analysed using Ig-coupled beads. Pristane-cyclodextrin complexes were used to study the effects of pristane in cultured cells.

Results 3 days after pristane application the proportion of cells positive for cleaved caspase 3 was highly increased in draining inguinal lymph nodes of DA.1F rats indicating apoptosis. Interestingly, the apoptosis rate decreased to normal levels as seen as in naive animals within 2 weeks but draining lymph nodes became extremely hypercellular. In peripheral blood both apoptosis and necrosis rates doubled during the first week after pristane injection. In the acute disease phase four times increased apoptosis and necrosis rates were observed which was in contrast to the draining lymph nodes which normalised during the disease course. The authors could not observe any phagoytosis deficiency in pristane-injected animals rather a strongly increased phagocytosis activity was seen in the acute disease phase. In line with this in vivo data, pristane induced pronounced apoptosis and secondary necrosis in a dose dependent manner when added to cultured rat splenocytes. 
Conclusion This data points toward a pronounced apoptosis/ necrosis inducing effect of pristane both in vitro and in vivo. This highly increased cell death might be the crucial initial trigger for the development of PIA. Thus, PIA appears to be an excellent model for studying very early immune processes, particularly the generation of neo-autoantigens, which might play a pivotal role in the pathogenesis of RA. 